\title{
Assessment of thrombin-activatable fibrinolysis inhibitor (TAFI) activation in acquired hemostatic dysfunction: a diagnostic challenge
}

\author{
Hyun Kyung Kim, M.D. \\ Department of Laboratory Medicine, Seoul National University College of Medicine, Seoul, Korea
}

Human thrombin activatable fibrinolysis inhibitor (TAFI) is a carboxypeptidase zymogen that was first described in 1995 [1]. It had been previously reported under different names such as carboxypeptidase basic (B), unstable (U), and arginine (R) [2]. The mature TAFI protein contains 401 amino acids, and after glycosylation, the molecular weight becomes $60 \mathrm{kDa}$. TAFI in its plasma zymogen form can be activated by thrombin, the thrombin-thrombomodulin complex, or plasmin. The activated TAFI (TAFIa) decays spontaneously into the inactive form of TAFI (TAFIai) through a temperature-dependent conformational change.

TAFIa plays a role in the inhibition of fibrinolysis by cleaving the carboxyl-terminal lysine residues from partially degraded fibrin. In the coagulation cascade, the generated thrombin can activate TAFI, and the resulting TAFIa inhibits fibrinolysis, finally resulting in the formation of more stable clots [3]. Therefore, a deficiency of the coagulation factors might reduce the TAFIa levels via reduced thrombin generation, resulting in the formation of an unstable clot. In an animal study, TAFI was activated during a thrombotic event, and the inhibition of TAFIa activity enhanced the efficacy of thrombolytic therapy, thereby confirming its role as an inhibitor of fibrinolysis [4-6]. TAFIa also plays a role in the modulation of inflammation through down-regulation of pericellular plasminogen activation and inactivation of the inflammatory peptides bradykinin and anaphylatoxins C3a and C5a. In mice with Escherichia coli-induced sepsis, TAFI deficiency prevented liver damage via inactivation of C3a and C5a [7].

The plasma concentration of TAFI appears to be under the control of genetic factors and non-genetic factors such as sex hormones, cytokines, and lipids [2]. Several diseases, including diabetes, kidney transplantation, hypertension, nephritic syndrome, insulin resistance, obesity, and inflammatory bowel disease, have been shown to be positively associated with plasma TAFI levels [2]. Because TAFI is expressed in adipocytes, an increased TAFI level can be expected in metabolic disturbance. The TAFI levels were also elevated in thrombotic diseases. In keeping with the role of TAFI as an antifibrinolytic factor, high plasma level of TAFI is a risk factor for thrombotic diseases. Meanwhile, TAFI levels are reduced in acquired hemostatic dysfunctions, such as post-partum hemorrhage and sepsis accompanying disseminated intravascular coagulation [3], because the enhanced generation of thrombin or plasmin may result in enhanced TAFI activation and consequent consumption of the TAFI zymogen.

Many different methods for measuring TAFI by using ELISA, enzymological methods, or functional methods have been described. All the methods have their inherent advantages and disadvantages, and to date, there is no reference standard for TAFI. Most studies have used the total antigen level of TAFI, but this has yielded ambiguous results, because of the existence of different isoforms and various degrees of activation. Ceres et al. [8] have reported the use of an ELISA that specifically detects TAFI activation. Since substantial TAFI activation occurs in acquired hemostatic dysfunction, ELISA that measures the extent of TAFI activation is potentially useful.

In this issue of the Korean Journal of Hematology, Park et al. [9] reported high levels of TAFI activation in sepsis patients by measuring TAFIa/ai levels using an ELISA that they developed "in-house." In the "in-house" ELISA, potato tuber carboxypeptidase inhibitor was used as a capture agent

This is an Open Access article distributed under the terms of the Creative Commons Attribution Non-Commercial License (http://creativecommons.org/licenses/by-nc/3.0) which permits unrestricted non-commercial use, distribution, and reproduction in any medium, provided the original work is properly cited. 
that specifically captured the activated and inactivated TAFI isoforms and did not capture the intact TAFI zymogen. Although only a few septic patients $(n=25)$ were enrolled in the study, the activation status of TAFI could clearly differentiate septic patients from healthy controls. This result is in agreement with the results of a recent study [10] that reported a significant elevation of TAFI activation in meningococcal infection. Since decreases in total TAFI level are associated with increases of TAFI activation level, simultaneous determination of total TAFI and TAFI activation marker is required to interpret the role of TAFI in acquired hemostatic dysfunctions including sepsis.

Although the principal physiologic role of TAFI is still a matter of debate, TAFI is reported to be an important protein in the fibrinolytic system. The advent of more accurate assays for measuring plasma TAFI levels may help validate the mechanism underlying TAFI activation in acquired hemostatic dysfunction. This information may help elucidate the contribution of the TAFI pathway to acquired hemostatic dysfunction and will form the basis of potential therapeutic strategies to manipulate the TAFI pathway for acquired hemostatic dysfunction.

\section{REFERENCES}

1. Bajzar L, Manuel R, Nesheim ME. Purification and characterization of TAFI, a thrombin-activable fibrinolysis inhibitor. J Biol Chem 1995;270:14477-84.

2. Boffa MB, Koschinsky ML. Curiouser and curiouser: recent advances in measurement of thrombin-activatable fibrinolysis inhibitor (TAFI) and in understanding its molecular genetics, gene regulation, and biological roles. Clin Biochem 2007;40:431-42.

3. Toh $\mathrm{CH}$. Characterization of thrombin activatable fibrinolysis inhibitor in normal and acquired haemostatic dysfunction. Blood Coagul Fibrinolysis 2003;14(Suppl 1):S69-71.

4. Redlitz A, Nicolini FA, Malycky JL, Topol EJ, Plow EF. Inducible carboxypeptidase activity. A role in clot lysis in vivo. Circulation 1996;93:1328-30.

5. Heylen E, Miljic P, Willemse J, et al. Procarboxypeptidase $\mathrm{U}$ (TAFI) contributes to the risk of thrombosis in patients with hereditary thrombophilia. Thromb Res 2009;124: 427-32.

6. Kraft P, Schwarz T, Meijers JC, Stoll G, Kleinschnitz C. Thrombin-activatable fibrinolysis inhibitor (TAFI) deficient mice are susceptible to intracerebral thrombosis and ischemic stroke. PLoS One 2010;5:e11658.

7. Renckens R, Roelofs JJ, ter Horst SA, et al. Absence of thrombin-activatable fibrinolysis inhibitor protects against sepsis-induced liver injury in mice. J Immunol 2005;175:6764-71.

8. Ceresa E, Brouwers E, Peeters M, Jern C, Declerck PJ, Gils A. Development of ELISAs measuring the extent of TAFI activation. Arterioscler Thromb Vasc Biol 2006;26:423-8.

9. Park R, Song J, An SS. Elevated TAFIa/ai levels in patient with sepsis and normal controls with TAFIa/ai specific ELISA. Korean J Hematol 2010;45:264-8.

10. Emonts M, de Bruijne EL, Guimarães AH, et al. Thrombinactivatable fibrinolysis inhibitor is associated with severity and outcome of severe meningococcal infection in children. J Thromb Haemost 2008;6:268-76. 